\title{
Abdominal Wall Endometriomas at Cesarean Section Scars: A Case Series
}

\author{
Kathryn Wampler*, Bailey Harwager, Kimberly Michael \\ University of Nebraska Medical Center, Omaha, NE, USA \\ Email: *kathryn.wampler@unmc.edu
}

How to cite this paper: Wampler, K., Harwager, B. and Michael, K. (2017) Abdominal Wall Endometriomas at Cesarean Section Scars: A Case Series. Open Journal of $\mathrm{Ob}$ stetrics and Gynecology, 7, 815-823. https://doi.org/10.4236/ojog.2017.78082

Received: June 15, 2017

Accepted: July 30, 2017

Published: August 2, 2017

Copyright @ 2017 by authors and Scientific Research Publishing Inc. This work is licensed under the Creative Commons Attribution International License (CC BY 4.0).

http://creativecommons.org/licenses/by/4.0/ (c) (i) Open Access

\begin{abstract}
Endometriosis, a common gynecological condition, is defined as the presence of functioning endometrial tissue outside the uterine cavity. It often presents as a cyclic, hormonally stimulated pain in women during their reproductive years. While endometriosis is usually located within the pelvis, it may also occur at intraperitoneal, intestinal, perineal, and distant ectopic sites. Although often diffuse, a localized, focal mass of endometrial tissue is termed as an endometrioma. In rare occurrences, an endometrioma will present superficially to the peritoneum within the abdominal wall following gynecologic or obstetric surgery. The presence of an abdominal wall endometrioma within a cesarean section scar may pose a diagnostic dilemma, which is often misdiagnosed, and results in surgery referrals for treatment. The clinical symptoms and sonographic appearance of abdominal wall endometriomas occurring at cesarean section scars are highlighted in this case series.
\end{abstract}

\section{Keywords}

Endometriosis, Endometrioma, Abdominal Wall Endometrioma, Cesarean Section Scar, Sonography

\section{Introduction}

Endometriosis is a common gynecological condition defined as the presence of functioning endometrial tissue outside the uterine cavity [1]. It often presents as a cyclic pain in women during their reproductive years that responds to hormonal stimulation and can lead to possible infertility [1]. About $10 \%-15 \%$ of women in their reproductive years will experience endometriosis [2]. Endometriosis occurs in both diffuse and localized forms. A focal mass of endometrial tissue is known as an endometrioma. Although the diagnosis of endometriosis is relatively common, the variable locations of endometrial implants may make it 
an uncommon differential diagnosis. Ectopic implantation of endometrial tissue is often discovered in the pelvic cavity on the surface of the peritoneum, ovaries, pouch of Douglas, and uterosacral ligaments [2]. Extrapelvic location of an endometrioma at intraperitoneal, intestinal, perineal, and distant ectopic sites occurs in only $1 \%-2 \%$ of patients [3]. While uncommon, endometriomas have been discovered in the peritoneum, bladder, kidneys, lungs, liver, gallbladder, bowel, central nervous system, umbilicus, and abdominal wall [1] [4].

The most common extrapelvic location for endometriosis is the abdominal wall, typically presenting within scars following gynecological or obstetric surgery [1]. Surgical scar endometriomas following a cesarean delivery are rare, with a prevalence estimated to be between $0.03 \%$ and $1 \%$ [1]. The rare presence of an abdominal wall endometrioma within a cesarean section scar may pose a diagnostic dilemma both clinically and with diagnostic imaging. Abdominal wall endometriosis is often misdiagnosed and the cause of surgery referrals for treatment [3]. Cesarean section scar endometriomas are often mistaken for pathologies such as an incisional hernia, lipoma, hematoma, abscess, or suture granuloma [5]. In conjunction with a clinical history, diagnosis of an abdominal wall endometrioma may be aided by utilization of diagnostic imaging modalities, including sonography, computed tomography (CT), and magnetic resonance imaging (MRI). Definitive diagnosis is usually made with histologic examination. The clinical symptoms and sonographic appearance of three cesarean section endometriomas occurring at the same institution are highlighted in this case series.

\section{Case Series}

\section{Case 1}

A G1P1 female in her mid-twenties presented with a painful and palpable lump located in her left lower quadrant. The patient first noticed the lump six months prior and could identify that the lump size and pain level increased with her menstrual cycles. Surgical history included an uncomplicated, low transverse $\mathrm{C}$-section three years prior. The patient denied any history of endometriosis, was otherwise healthy with no significant medical history.

A transabdominal pelvic sonogram was performed with detailed examination of the abdominal wall at the area of pain utilizing a Siemens Acuson S2000, with a C4-1 $\mathrm{MHz}$ curved transducer and $\mathrm{L} 49-4 \mathrm{MHz}$ linear transducer under the general gynecologic setting. The uterus and ovaries were unremarkable. Assessment of left lower quadrant abdominal wall showed an oval, well circumscribed, hypoechoic mass with smooth borders. Small cystic spaces were also noted. The mass was located anterior to the rectus abdominus muscle within the subcutaneous tissue (Figure $1(\mathrm{~A})$ ). The mass measured $1.5 \times 1.3 \times 1.0 \mathrm{~cm}$ and was hypovascular by color Power Doppler imaging. (Figure 1(B)).

An MRI of the abdomen with contrast confirmed the mass was in the deep subcutaneous fat of the left lower quadrant abdominal wall, just superficial to the rectus sheath. (Figure $1(\mathrm{C})$ ). Following the MRI, an ultrasound guided percuta- 

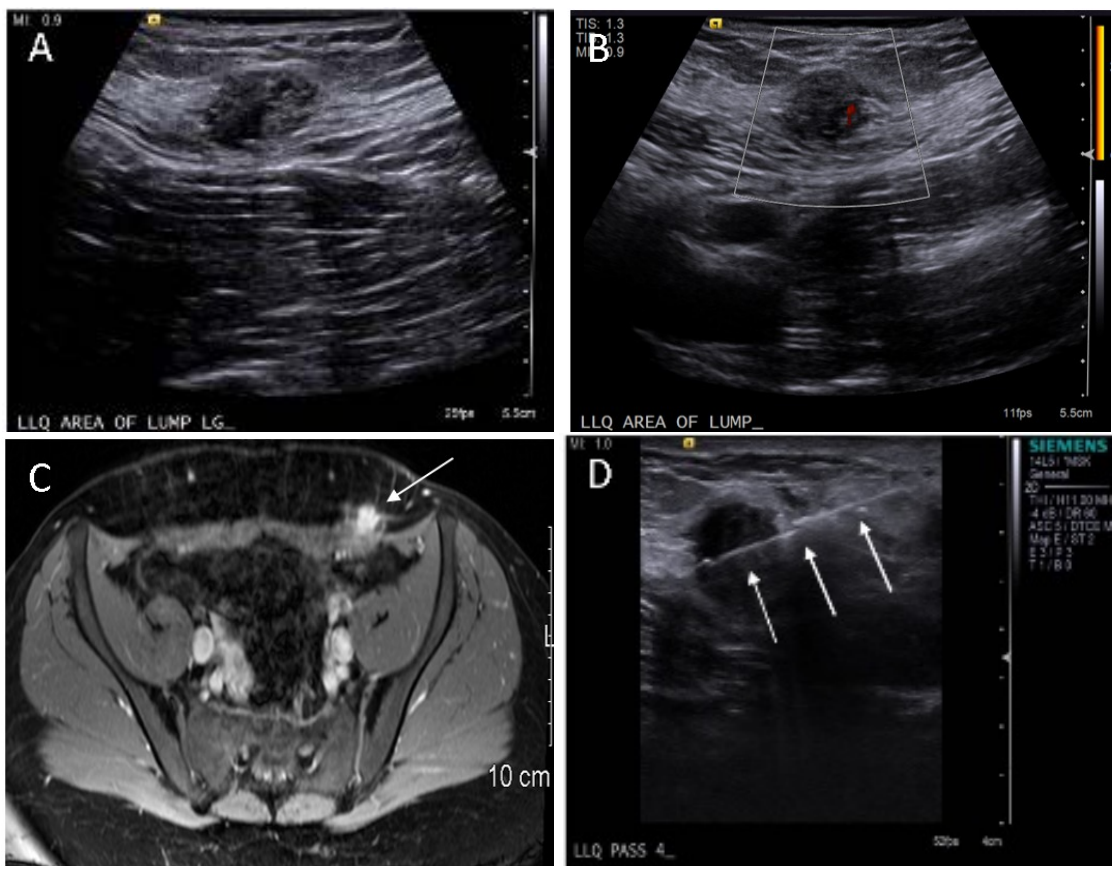

Figure 1. (A) Longitudinal sonographic demonstration of the abdominal wall mass; (B) Sonographic demonstration of the mass in a transverse plane. The mass is hypoechoic and contais small cystic spaces; (C) A T1 fat suppressed axial MRI image demonstrating the enhancing mass located within in the deep subcutaneous fat of the left lower quadrant abdominal wall (arrow); (D) Sonographic image demonstrating a core biopsy of the mass. Notice the "bayonet artifact" (arrows) created as the needle passes through subcutaneous tissue and into the mass.

neous core needle biopsy was performed (Figure 1(D)). Biopsy results diagnosed an endometrioma located within a post cesarean section scar. The patient did not return for further treatment.

\section{Case 2}

A G2P2 female in her early thirties presented with a focal painful swelling in her suprapubic area present for approximately three weeks. In addition to the painful lump, she also reported some discomfort while urinating, but had no change in bowel function. Her menstrual cycles were regular, but associated with mild dysmenorrhea. Surgical history included an uncomplicated, low transverse cesarean section five years prior. The patient denied any history of endometriosis and had a normal pelvic ultrasound four years earlier. The patient's clinician ordered a pelvic ultrasound to evaluate the lump; most likely an endometrioma at the site of her cesarean scar.

Sonographic evaluation of the pelvis was performed using a Philips iU22 with a C5-1 MHz curved transducer and L12-5 MHz linear transducer under the general gynecologic setting. The sonographic report described the uterus and ovaries as unremarkable. An oval, well circumscribed, hypoechoic, suprapubic mass was identified anterior to the bladder. It was not visible on the prior sonogram (Figure 2(A) and Figure 2(B)). Multiple small cystic areas were visualized within the $2.0 \mathrm{~cm}$ mass located in the suprapubic rectus abdominus muscle 

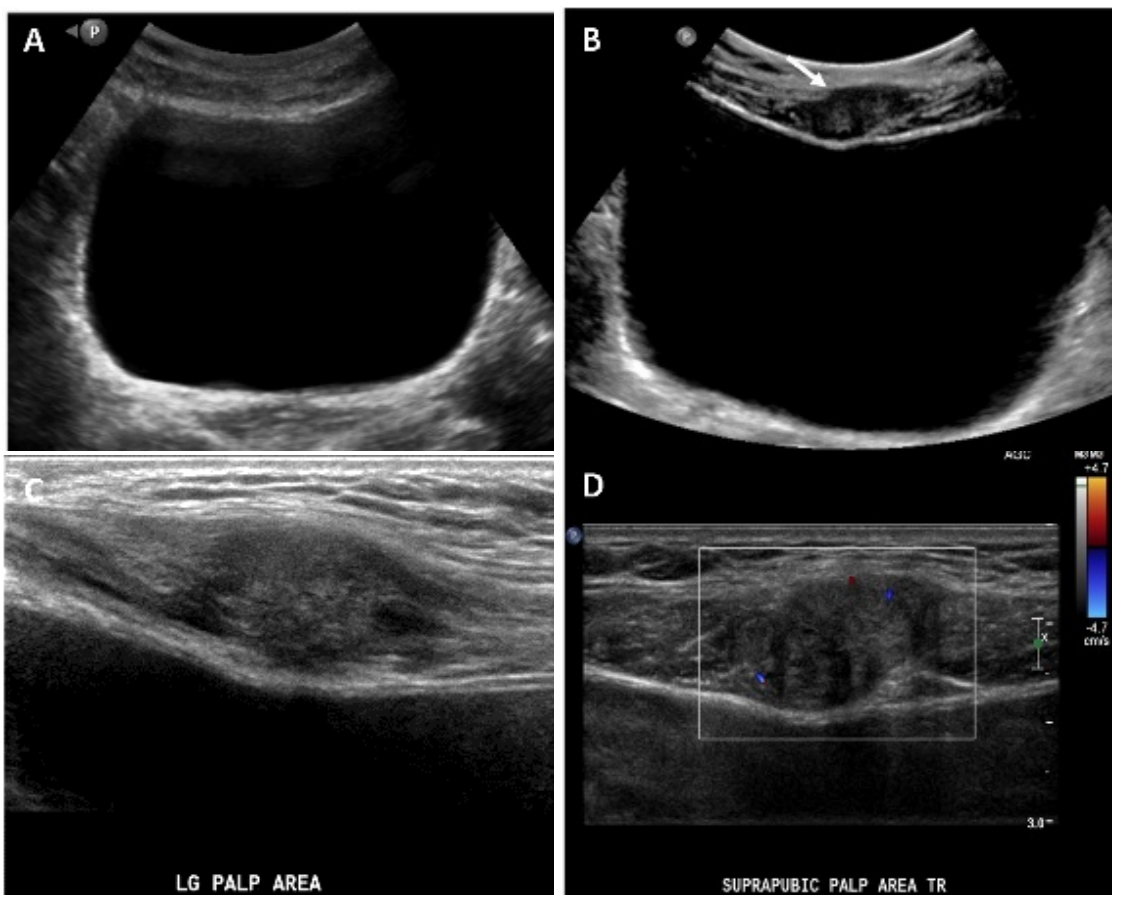

Figure 2. (A) Transabdominal sonographic image demonstrating the patient's normal abdominal wall four years prior to presenting with a suprapubic lump; (B) Transabdominal sonographic image demonstrating a hypoechoic, suprapubic mass (arrow) anterior to the bladder, which was not visible on the prior sonogram (Figure 2(B)); (C) Sonographic image of the suprapubic mass within the rectus abdominus muscle; (D) Vascularity was demonstrated within the mass with use of color Doppler.

(Figure 2(C)). Vascularity was demonstrated within the nodule using a low PRF color Doppler scale (Figure 2(D)). Correlation with patient's surgical history and clinical symptoms, suggested the mass was most likely an endometrioma.

The patient was offered medical management with oral contraceptives, but opted for Depo-Provera. Follow up three months later the patient reported a significant improvement in her pain.

\section{Case 3}

A G6P6 female in her early forties presented with a painful, palpable lump located in the left lower quadrant near her recent cesarean section scar. Surgical history included two previous C-sections and removal of a $3.9 \mathrm{~cm}$ abdominal wall endometrioma following her first cesarean section. The patient's symptoms were similar to those associated with her previous endometrioma, including cyclical pain related to her menstrual cycle.

Sonographic evaluation of the abdominal wall was performed utilizing a Philips iU22 with a L12-5 MHz linear transducer under the general abdominal setting. A well circumscribed, hyperechoic mass involving the fascia and rectus abdominus muscle was identified within the left lower quadrant (Figure 3(A)). The smooth walled mass (arrow) measured approximately $3.0 \mathrm{~cm}$ in the longest dimension and was within proximity to the recent cesarean section scar (Figure 3(B)). Multiple small cystic spaces were also noted internally. High resistance, 

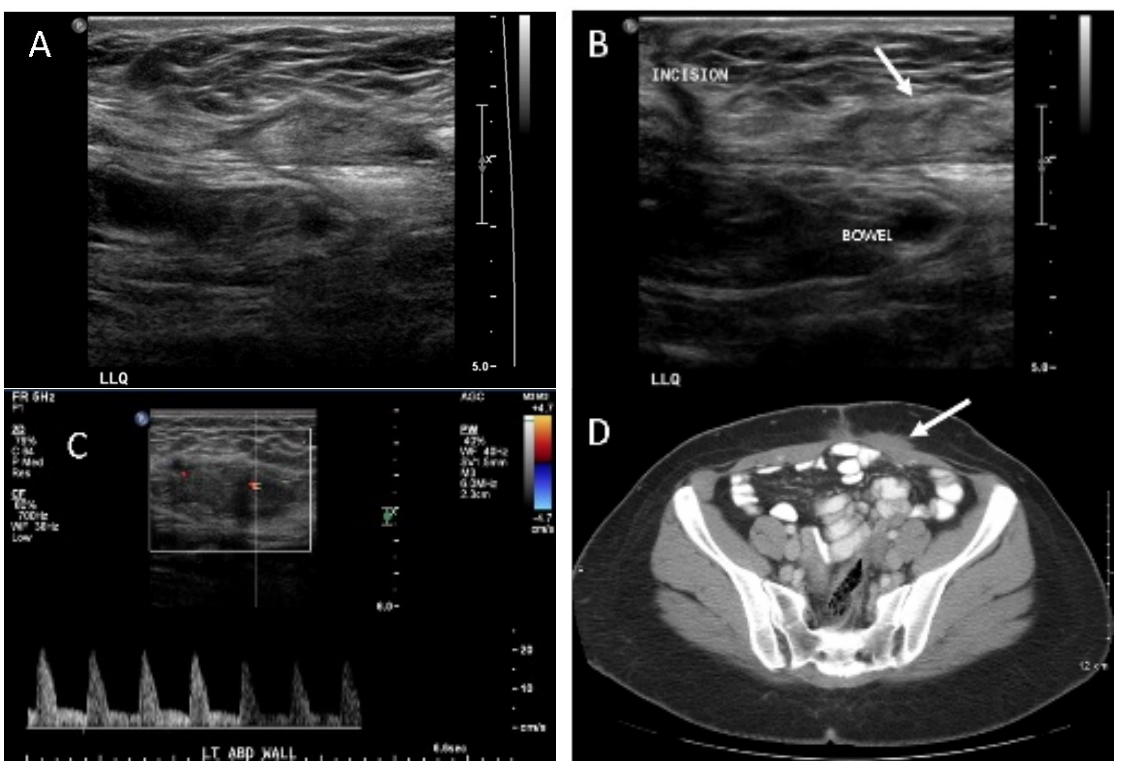

Figure 3. (A) Sonographic image of left lower quadrant abdominal wall mass involving the fascia and rectus abdominus muscle. The mass is hypoechoic and contains small cystic spaces; (B) Sonographic image of the mass (arrow) demonstrating proximity to the recent cesarean section scar; (C) Spectral tracing demonstrating arterial vascularity within the mass; (D) Axial CT image of the pelvis demonstrating an abdominal wall mass in the left lower quadrant (arrow).

low velocity arterial flow was documented within the mass by color Doppler (Figure $3(\mathrm{C})$ ). With the patient's clinical symptoms, history of recent cesarean section and prior endometrioma, the mass was most likely an endometrioma.

The patient opted for medical therapy and continued use of oral contraceptives for four months. The patient returned at that time with increased pelvic and abdominal wall pain. A CT of the pelvis was normal, but again demonstrated the left lower quadrant abdominal wall endometrioma (Figure 3(D)). Ultimately, the patient elected to have a total abdominal hysterectomy. The abdominal wall mass was removed during the hysterectomy, which was confirmed by pathology to be an endometrioma.

\section{Discussion}

Endometriomas within scar tissue are rare and often difficult to diagnose. Clinicians should consider the following differential diagnoses for a mass located within a surgical scar: an incisional hernia, lipoma, hematoma, abscess, suture granuloma, and endometrioma [5]. While abdominal wall endometriomas are often misdiagnosed, it must be considered when a woman in her reproductive years presents with a post-operative abdominal wall mass [6]. While the use of sonography does not always lead to a specific diagnosis of an abdominal wall endometrioma, several consistent sonographic findings were documented in this case series. The endometriomas of note were all well-circumscribed, oval, discrete masses. The masses were predominantly hypoechoic and contained small anechoic, or cystic spaces. Locations of the endometriomas were all within the 
abdominal wall in proximity to prior cesarean section scars. Color and spectral Doppler evaluation demonstrated vascularity within the endometriomas. The patients in this case series presented with similar complaints of a painful, palpable abdominal wall mass and history of prior cesarean section. Two of the three reported cyclical pain associated with menstruation.

A study conducted by Francica et al. examined twelve cases of endometriomas within cesarean section scars and characterized the sonographic findings. This study concluded that the sonographic appearance of cesarean section endometriomas include a discrete subcutaneous, hypoechoic nodule, scattered with hyperechoic strands. Margins of the endometrioma were often noted to be irregular and speculated, infiltrating the abdominal muscular fascia, and circumscribed by a hyperechoic ring. Endometriomas larger than $3 \mathrm{~cm}$ demonstrated small cystic areas, as well as the loss of the well-circumscribed oval shape. Additionally, a vascular pedicle with arterial flow was seen entering the nodule with the use of color Doppler interrogation. It was noted that as endometriomas increase size, vascularization is more visible. Masses smaller than $1.5 \mathrm{~cm}$ demonstrated less vascularity than larger masses [4]. In this study, sonography was the sole diagnostic imaging modality utilized for diagnose and continued evaluation of the endometriomas.

CT and MRI may also be utilized to image endometriomas, although results are often nonspecific and may not contribute any additional information to a thorough sonographic examination [4]. CT and MRI scans both demonstrate endometriomas as a solid, enhancing mass within the abdominal wall, and may be used in preoperative planning [7]. It has been reported that with the use of sonography and color Doppler, in addition to the clinical data, a reliable pre-operative diagnosis may be reached [1]. A biopsy, however, should always be performed following imaging studies if the results are nonspecific [2]. Cytology smears will show epithelial cells, stromal cells, and macrophages, although the presence of two of the three is required to diagnose endometriosis [8].

While endometriosis is typically located within the pelvis, the occurrence of extrapelvic endometriosis should not be overlooked. Although rare, extrapelvic endometriomas are most commonly found in the abdominal wall within a caesarean section scar [1]. Abdominal wall endometriomas may be contained in the subcutaneous tissues, the rectus abdominus muscle, or involve both [9]. Only $14 \%-26 \%$ of women that experience scar endometriosis have concomitant pelvic endometriosis [9]. A tender abdominal wall mass that causes cyclic pain should be considered suggestive of an abdominal wall endometrioma in females with a previous history obstetric or gynecologic surgery [3]. The clinical presentation of an abdominal wall endometrioma is typically a painful, often palpable, subcutaneous mass near surgical scars in parous women who also have a previous history of gynecological or obstetrical surgery [6]. A study conducted by Horton et al. reviewed twenty-nine articles describing 455 patients with abdominal wall endometriomas. The mean age at diagnosis was found to be 31.4 years. It has been identified through various studies that $75 \%$ of symptomatic 
women are between the ages of 25 and 45 years, leading to the conclusion that endometriosis usually occurs in women during their reproductive years [6]. The Horton study found that abdominal wall endometriosis presented as a palpable mass in $96 \%$ of cases and $87 \%$ presented with pain [5]. The pain associated with the palpable mass was found to fluctuate with the menstrual cycle in $57 \%$ of cases [5]. The duration of the symptoms has been found to vary from months to several years [3]. A mean of 3.6 years has been given to account for onset of symptoms following the patients last gynecological or obstetrics surgery [5]. Literature indicates that the mean size of an endometrioma is $3.1 \mathrm{~cm}(1.5-4.8$ range) [1].

The leading theory to explain the pathogenesis of abdominal wall endometriosis is that, during a surgery or procedure that opens the uterus, endometrial cells are transported during and transplanted to an ectopic location [6] [10]. Cesarean sections are proven to be a risk factor for acquiring an abdominal wall endometriomas [3]. It has been hypothesized that postoperative occurrence of an endometrioma in the incisional scar may increase with failure to close both the parietal and visceral peritoneum with sutures during caesarean section [2]. In clinical practice, the occurrence of endometriomas has been documented in incisions with possible contact with endometrial tissue, including cesarean section and hysterectomy [6]. Although abdominal wall endometriomas occur most commonly after operations on the uterus and fallopian tubes, it has also been reported with other surgical procedures including appendectomy, episiotomy, laparoscopic procedure, amniocentesis, and inguinal herniorrhaphy [6] [11]. The diagnosis of extrapelvic endometriomas has proven that endometrial tissue may be transplanted and survive at ectopic locations [1].

Medical therapy is commonly used to relieve the clinical symptoms of endometriomas. Hormone suppression slows down the regulation of the hypothalamus-pituitary-ovarian hormonal axis, although rarely does complete regression of endometriosis occur with medical therapy alone [1] [3]. If hormone therapy does not influence the endometrioma, surgical removal may be required [3]. To prevent local reoccurrence of endometriomas and tissue spread of endometriosis, a wide incision with clear margins is the treatment of choice when surgical removal is implemented [6] [8]. Following surgical excision, endometriomas rarely reoccur [12].

\section{Conclusion}

Endometriosis is the presence of functioning endometrial tissue outside the uterine cavity [1]. An endometrioma is a well-circumscribed mass of endometrial tissue, which may be located within the pelvis or extrapelvically [6]. Subcutaneous endometriomas near cesarean section scars are a rare form of extrapelvic endometriosis, occurring less than $1 \%$ of all women who have had cesarean sections [4]. The typical clinical presentation of an abdominal wall endometrioma is a palpable nodule near a surgical scar that is accompanied by cyclic pain during menses; correlating with the female hormone levels [4]. In women who present 
with painful nodules near cesarean section scars, along with cyclic or continuous lower abdominal pain, careful sonographic exploration of the abdominal wall is recommended [4]. This case series demonstrates the patient symptoms and sonographic findings of abdominal wall endometriomas related to cesarean section scars. Findings include a discrete, oval hypoechoic mass with hyperechoic or cystic internal components, and vascularity with Doppler interrogation. With correlation of surgical and gynecological histories, through sonographic imaging, and biopsy of the nodule if necessary, treatment can be attained [6]. Medical treatment for abdominal wall endometriomas may decrease symptoms, but surgical removal may be required if symptoms progress [1].

\section{References}

[1] Sengul, I., Sengul, D., Kahyaoglu, S. and Kahyaoglu, I. (2009) Incisional Endometriosis: A Report of 3 Cases. Canadian Journal of Surgery, 52, 444-445.

[2] Neri, I., Tabanelli, M., Dika, E., Valeria, G. and Patrizi, A. (2007) Diagnosis and Treatment of Post-Caesarean Scar Endometriosis. Acta Dermato-Venereologica, 87, 428-429. https://doi.org/10.2340/00015555-0269

[3] Oh, E.M., Lee, W., Kang, J.M., Choi, S.T., Kim, K.K. and Lee, W.K. (2014) A Surgeon's Perspective of Abdominal Wall Endometriosis at a Caesarean Section Incision: Nine Cases in a Single Institution. Surgery Research and Practice, 2014, 1-4. https://doi.org/10.1155/2014/765372

[4] Francica, G., Giardiello, C., Angelone, G., Cristiano, S., Finelli, R. and Tramontano, G. (2003) Abdominal Wall Endometriomas near Caesarean Delivery Scars. The American Institute of Ultrasound Medicine, 22, 1041-1047. https://doi.org/10.7863/jum.2003.22.10.1041

[5] Horton, J., DeZee, K., Ahnfeldt, E. and Wagner, M. (2008) Abdominal Wall Endometriosis: A Surgeon's Perspective and Review of 445 Cases. The American Journal of Surgery, 196, 207-212. https://doi.org/10.1016/j.amjsurg.2007.07.035

[6] Dwivedi, A.J., Agrawal, S.N. and Silva, Y.J. (2002) Abdominal Wall Endometriomas. Digestive Diseases and Sciences, 47, 456-461. https://doi.org/10.1023/A:1013711314870

[7] Hensen, J., Van Breda Vriesman, A. and Puylaert, J. (2006) Abdominal Wall Endometriosis: Clinical Presentation and Imaging Features with Emphasis on Sonography. American Journal of Roentgenology, 168, 616-620. https://doi.org/10.2214/AJR.04.1619

[8] Veda, P. and Srinivasaiah, M. (2010) Incisional Endometriosis: Diagnosed by Fine Needle Aspiration Cytology. Journal of Laboratory Physicians, 2, 117-120. https://doi.org/10.4103/0974-2727.72216

[9] Aydin, O. (2007) Scar Endometriosis: A Gynecologic Pathology Often Presented to the General Surgeon Rather than the Gynecologist. Langenbeck's Archives of Surgery, 392, 105-109. https://doi.org/10.1007/s00423-006-0107-8

[10] Gidwaney, R., Badler, R., Yam, B., et al. (2012) Endometriosis of Abdominal and Pelvic Wall Scars: Multimodality Imaging Findings, Pathologic Correlation, and Radiologic Mimics. RadioGraphics, 32, 2031-2043. https://doi.org/10.1148/rg.327125024

[11] Al-Jabri, K. (2009) Endometriosis at Caesarian Section Scar. Oman Medical Journal, 24, 294-295. https://doi.org/10.5001/omj.2009.59 
[12] Pados, G., Tympanidis, J., Zafrakas, M., Athanatos, D. and Bontis, J. (2008) Ultrasound and MR Imaging in Preoperative Evaluation of Two Rare Cases of Scar Endometriosis. Cases Journal, 1, 97. https://doi.org/10.1186/1757-1626-1-97

Submit or recommend next manuscript to SCIRP and we will provide best service for you:

Accepting pre-submission inquiries through Email, Facebook, LinkedIn, Twitter, etc. A wide selection of journals (inclusive of 9 subjects, more than 200 journals)

Providing 24-hour high-quality service

User-friendly online submission system

Fair and swift peer-review system

Efficient typesetting and proofreading procedure

Display of the result of downloads and visits, as well as the number of cited articles Maximum dissemination of your research work

Submit your manuscript at: http://papersubmission.scirp.org/

Or contact ojog@scirp.org 